\title{
Kreuth V initiative: European consensus proposals for treatment of hemophilia using standard products, extended half-life coagulation factor concentrates and non-replacement therapies
}

Haematologica 2020

Volume 105(8):2038-2043

\section{Correspondence: \\ FLORA PEYVANDI, \\ flora.peyvandi@unimi.it \\ Received:November 18, 2019. \\ Accepted: May 20, 2020. \\ Pre-published: May 28, 2020.}

doi:10.3324/haematol.2019.242735

Check the online version for the most updated information on this article, online supplements, and information on authorship \& disclosures: www. haematologica.org/content/105/8/2038

\section{(C)2020 Ferrata Storti Foundation}

Material published in Haematologica is covered by copyright. All rights are reserved to the Ferrata Storti Foundation. Use of published material is allowed under the following terms and conditions:

https://creativecommons.org/licenses/by-nc/4.0/legalcode. Copies of published material are allowed for personal or internal use. Sharing published material for non-commercial purposes is subject to the following conditions:

https://creativecommons.oró/licenses/by-nc/4.0/legalcode, sect. 3. Reproducing and sharing published material for commercial purposes is not allowed without permission in writing from the publisher.
Flora Peyvandi, ${ }^{1,2}$ Karin Berger, ${ }^{3,4}$ Rainer Seitz, ${ }^{5}$ Anneliese Hilger, ${ }^{5}$ Marie-Laure Hecquet, ${ }^{6}$ Michael Wierer, ${ }^{6}$ Karl-Heinz Buchheit, ${ }^{6}$ Brian O'Mahony, ${ }^{7,8}$ Amanda Bok, ${ }^{8}$ Mike Makris, ${ }^{9}$ Ulrich Mansmann, ${ }^{4}$ Wolfgang Schramm ${ }^{10}$ and Pier Mannuccio Mannucci ${ }^{1}$

${ }^{1}$ Fondazione IRCCS Ca' Granda Ospedale Maggiore Policlinico, Angelo Bianchi Bonomi Hemophilia and Thrombosis Center and Fondazione Luigi Villa, Milan, Italy; ${ }^{2}$ Università degli Studi di Milano, Department of Pathophysiology and Transplantation, Milan, Italy; ${ }^{3}$ University Hospital, Ludwig-Maximilian University, Department of Medicine III, Munich, Germany; ${ }^{4}$ Institute for Medical Information Processing, Biometry and Epidemiology (IBE), Ludwig-Maximilian-University, Munich, Germany; ${ }^{5}$ Paul-Ehrlich-Institut, Langen, Germany; ${ }^{6}$ European Directorate for the Quality of Medicines and Healthcare, Strasbourg, France; ${ }^{7}$ Trinity College, Dublin, Ireland; ${ }^{8}$ European Haemophilia Consortium, Brussels, Belgium; ${ }^{9}$ Sheffield Haemophilia and Thrombosis Centre, Sheffield, UK and ${ }^{10}$ Department of Transfusion Medicine and Haemostasis, Ludwig-Maximilians-University, Munich, Germany

\section{ABSTRACT}

his report contains the updated consensus recommendations for optimal hemophilia care produced in 2019 by three Working Groups (WG) on behalf of the European Directorate for Quality of Medicines and Healthcare in the frame of the Kreuth V Initiative. WG1 recommended access to prophylaxis for all patients, the achievement of plasma factor trough levels of at least 3-5\% when extended half-life factor VIII (FVIII) and FIX products are used, a personalized treatment regimen, and a choice of chromogenic assays for treatment monitoring. It was also emphasized that innovative therapies should be supervised by hemophilia comprehensive care centers. WG2 recommended mandatory collection of postmarketing data to assure the long-term safety and efficacy of new hemophilia therapies, the establishment of national patient registries including the core data recommended by the European Medicines Agency and the International Society on Thrombosis and Haemostasis, with adequate support under public control, and greater collaboration to facilitate a comprehensive data evaluation throughout Europe. WG3 discussed methodological aspects of hemophilia care in the context of access decisions, particularly for innovative therapies, and recommended that clinical studies should be designed to provide the quality of evidence needed by regulatory authorities, HTA bodies and healthcare providers. The dialogue between all stakeholders in hemophilia care and patient organizations should be fostered to implement these recommendations.

\section{Introduction}

The Wildbad Kreuth Initiative started in 1999 with a seminar including experts from 15 European Community member states. This was followed over the next few years by a series of four meetings. Treatment of hemophilia has always been the focus of the initiative, given the increasing number of diagnosed patients and the importance of providing them with optimal therapies. The objectives of the Initiative were to evaluate the state of hemophilia therapy, identify areas in need of further studies, and provide updated recommendations for optimal use of blood 
products for treatment. The first Kreuth meeting also dealt with the optimal use of the available products in hemophilia therapy, emphasizing that the main priority was the safety of blood and blood products. Attention was also given to the need to guarantee an effective treatment, ensuring that all subjects with coagulation disorders can benefit from these lifesaving therapies. ${ }^{1}$

The following Kreuth meetings were periodically convened under the joint auspices of the Ludwig-Maximilian University of Munich (LMU), the Paul Ehrlich Institute (PEI), and the Council of Europe through its European Directorate for the Quality of Medicines and Healthcare (EDOM), under the aegis of the European Committee on Blood Transfusion (CD-P-TS). The latter institution provides resolutions that are non-binding but that are still strong indications for member states. The second Kreuth meeting in 2009 was attended by 110 transfusion medicine experts, hemophilia clinicians and regulatory authority representatives from 38 countries. New recommendations were provided regarding the best clinical practice on hemophilia, home treatment, genetic counselling and equal treatment across European member states. ${ }^{2}$

The following two meetings in 2013 and 2016 focused on the optimal use of coagulation factors and provided the opportunity to review trends in the use of factor concentrates. $^{3,4}$ A total of 12 recommendations were made in 2016, dealing with national protocols or guidelines for the management of aging patients with hemophilia, the minimum utilization of FVIII and FIX concentrates in each country, treatment for hepatitis C with direct-acting antiviral agents, genotype analysis for all patients with severe hemophilia, access to bypassing agents and immune tolerance for those with inhibitors, individualization of treatment regimens with extended half-life products and the attainment of the highest possible rate of bleeding prevention by increasing the trough plasma factor levels. The recommendations emerging from the 2013 and 2016 Kreuth meetings were subsequently incorporated by the EDQM into proposals for resolutions adopted by the Committee of Ministers of the Council of Europe, with the objective of increasing their visibility and providing official support. The 2017 Resolution [Resolution $\mathrm{CM} / \operatorname{Res}(2017) 43$ on principles concerning hemophilia therapies $]^{5}$ listed 17 principles.

The 2019 meeting was the most recent opportunity for the official delegates nominated by 26 Council of Europe members and observer states, along with members from the academia, the European Hemophilia Consortium (EHC) and the European Medicines Agency (EMA), to review trends in the use of standard half-life coagulation factor concentrates, but also of the new extended half-life products and non-replacement therapies.

\section{Methodology}

The "Wildbad Kreuth Initiative V - Optimal Treatment of hemophilia symposium" that took place in June 2019 involved clinicians, regulators and patient organizations from 26 European countries.

The participants were experts invited by the Scientific Programme Committee, as well as delegates appointed by the Council of Europe (CoE) Member States on the invitation of the EDOM, plus delegates from the patient organizations, the European Hemophilia Consortium (EHC) and World Federation of Hemophilia (WFH), and the European Medicines Agency (EMA). Several of the participating experts are active members of the European Association for Hemophilia and Allied Disorders (EAHAD) or other scientific societies. Industry representatives had the opportunity to participate in the open sessions, but were excluded from the discussion and formulation of the recommendations.

The topics of the symposium were defined by the Scientific Committee on the basis of the results and recommendations of the previous symposia and the latest developments in the field of therapies for hemophilia. During the subsequent open plenary sessions, the invited experts presented an overview of the current state-of-theart on hemophilia therapy in Europe and treatment progress on the topics predefined by the Scientific Committee. Their solid scientific background and indepth knowledge of the particular situation of hemophilia treatment in their own countries enabled the chosen delegates taking part to elaborate the recommendations to be presented to the Health Authorities of the CoE Member States.

The experts met in three different working groups to discuss and develop the new recommendations. Each working group was responsible for one of the following areas of discussion:

- clinical evaluation of hemophilia therapy;

- collection of data on hemophilia therapy;

- methodological aspects of hemophilia therapy.

Each working group prepared an interim report which was then discussed with the general assembly. If the report received full and unanimous consensus, it would then go forward for final approval. Based on the final report, this manuscript was prepared and circulated among all participants of all three working groups. Literature research was based on articles published in peer reviewed journals. Medline and PubMed were also searched for all articles published in English in the last ten years. Furthermore, data were extracted from the abstracts of the more recent international congresses.

\section{Recommendation 1}

Prophylactic treatment should be available to all hemophilia patients, with or without inhibitors, and access to physiotherapy should be provided.

Prophylaxis in hemophilia is considered the standard of care to prevent joint bleeding and related arthropathy, with the objective of preserving a normal musculoskeletal function. Manco-Johnson established the superiority of prophylactic versus on-demand therapy in a randomized clinical trial in 2007. ${ }^{6}$ Primary prophylaxis in hemophilia should start at a very young age ( $\leq 2$ years of age) before joint disease develops, and usually requires coagulation factor infusions 2-3 times per week, whereas secondary prophylaxis begins after the onset of joint disease. ${ }^{7}$ There are many prophylactic schedules, although the optimum dosing regimen is still to be defined. Prophylaxis with standard half-life coagulation factor products is usually given at a dose of $25-40 \mathrm{IU} / \mathrm{kg} \mathrm{2-3}$ times per week, ${ }^{7,8}$ whereas with the extended half-life products, prophylaxis regimens with intervals of 3-5 days in hemophilia $A$ and once every 7-14 days in hemophilia B can be effectively implemented. ${ }^{9-11}$

Currently, the availability of a non-replacement therapy administered subcutaneously such as emicizumab makes 
prophylaxis accessible to all patients with hemophilia A, including those with FVIII inhibitors, and this may help to reduce bleeding events and improve quality of life. . $^{12,13}$

For optimal care of hemophilia, the multidisciplinary team of specialists should include physiotherapists who should be involved from diagnosis and deal with the functional recovery after each musculoskeletal bleeding. They should also provide a rehabilitation program tailored to tackle all the problems related to this chronic condition. ${ }^{14}$

\section{Recommendation 2}

With increased treatment options, appropriate instruments should be developed to personalize treatment regimens for all patients with hemophilia $A$ and $B$.

The aim of prophylaxis is to minimize or abolish bleeding events, and thus improve quality of life in patients. Prophylaxis regimens with standard and extended half-life products have been shown to be effective at preserving joint function and preventing bleeding episodes, although significant variability was seen among individuals exposed to the same treatment regimen. ${ }^{15}$ Inter- and intraindividual variability in coagulation factor pharmacokinetics is thought to be the main determinant of uncertainty in the standardization of prophylaxis regimens. Appropriate tools must be developed to personalize treatment regimens, taking into account each patient's individual lifestyle and pharmacokinetic profile. ${ }^{16}$ The individualization of prophylaxis is the best strategy to improve patients' quality of life with the ambitious goal of zero bleeding in the near future.

\section{Recommendation 3}

With extended half-life therapies, a minimum trough level of 3-5\% should be achieved to preserve joint status.

Patients with severe hemophilia suffer from repeated and prolonged spontaneous bleeding episodes, mainly in muscles and joints, that result in disabling musculoskeletal damage and chronic arthropathy. The aim of prophylaxis in hemophilia is to reduce the risk of bleeding in order to preserve normal musculoskeletal function. Prophylaxis dosing regimens using standard half-life FVIII and FIX products can achieve trough plasma levels of $1-2 \%,{ }^{17}$ but the introduction of extended half-life products significantly improves efficacy by achieving higher trough levels. A further improvement due to the forthcoming second-generation extended half-life products and gene therapies might lead to a further increase in the achievement of almost normal trough factor levels. For the time being, with the opportunities provided by the availability of extended half-life products, the aim should be to achieve a minimum trough level of $3-5 \%$ in order to preserve joint function.

\section{Recommendation 4}

For the post infusion measurement of extended half-life products chromogenic assays should be used.

Hemophilia patients must be monitored by laboratory testing in order to assess the optimal plasma factor levels after concentrate infusion. One-stage clotting or chromogenic substrate assays have been used for monitoring post-infusion levels. However, with the introduction of extended half-life products, discrepancies between onestage and chromogenic assays have been observed. The choice of APTT reagents in the one-stage assays, and particularly the source of the contact activator, can influence assay sensitivity to the extended half-life products. This may lead to factor levels in patients being under- or overestimated according to the assay used for monitoring, with a potential negative effect on management. Therefore, experts recommended the adoption of a practical approach of switching from one-stage clotting assays to chromogenic assays. ${ }^{18,19}$

\section{Recommendation 5}

When using non-replacement therapies, for example for patients on emicizumab, some laboratory issues should be considered to correctly measure the procoagulant activity, FVIII levels after infusion of FVIII concentrate and in the estimation of FVIII inhibitors.

Novel non-replacement therapies that reduce the effect of natural anticoagulants rather than replace the deficient factor have been developed. One approach is based upon the use of a monoclonal antibody (concizumab) against tissue factor pathway inhibitor (TFPI); another emerging class is based on small interference RNA (siRNA) that reduces antithrombin expression. A different approach is represented by a bispecific antibody (emicizumab) that mimics the co-factor function of FVIII by bridging FIXa and FX. Special consideration should be given to laboratory monitoring in patients on the aforementioned novel approaches, especially for emicizumab, since this is the first non-replacement drug approved by both European and US medicine regulatory agencies (the EMA and the US Food and Drug Administration) for prophylaxis in adult and pediatric patients with hemophilia A, with and without inhibitors, and is currently used in clinical practice.

Emicizumab does not affect the prothrombin and thrombin time, but tests based on intrinsic coagulation are affected by this drug. ${ }^{19,20}$ Conventional one-stage clotting assay over-estimates the procoagulant activity of emicizumab, so that a modified FVIII one-stage clotting assay should be used to monitor this activity. The chromogenic FVIII assay is sensitive to emicizumab and provides an indirect measure of the procoagulant activity and drug concentration when reagents of human origin are used. However, there is no evidence that the procoagulant activity or emicizumab concentrations are correlated with its hemostatic efficacy when assessed by the chromogenic assay employing human reagents. The measurement of plasma FVIII levels after in vitro addition of FVIII concentrate and the measurement of anti-FVIII inhibitor titer by the conventional Bethesda method employing human reagents are affected by the drug in treated patients. Therefore, in order to measure post-infusion FVIII levels, and accurately detect the inhibitor titer, a chromogenic assay can be used, but only with reagents of bovine origin that are insensitive to the presence of the drug. ${ }^{19-23}$

\section{Recommendation 6}

The management of patients with hemophilia, particularly those using non-replacement therapies and gene therapies, should be supervised by Comprehensive Care Centres, such as the certified European Haemophilia Comprehensive Care Centres (EHCCC).

Hemophilia patients with and without inhibitors in Europe will be using more and more novel subcutaneous and innovative non-replacement therapies such as emicizumab. Hemophilia specialists must manage these patients in comprehensive care centers, particularly in the event of major intercurrent bleeds or surgery, because the physicians involved must have sufficient knowledge of 
the novel drugs to be able to handle any side effect. The need for additional hemostatic drugs, such as bypassing agents, at the time of breakthrough bleeding and surgery may increase the risk of thrombosis, and this must be carefully evaluated. In general, patients should be managed by EHCCC. The future objective of these expert centers should be the preparation and standardization of specific healthcare packages integrating comprehensive procedures for the management of hemophilia patients (with and without inhibitors) using emicizumab and other novel drugs during prophylaxis, with or without the addition of other hemostatic drugs during intercurrent bleeds or at the time of a surgery. The European Association for Hemophilia and Allied Disorders (EAHAD) and the European Hemophilia Consortium (EHC) need to update their joint European certification system, moving from the current self-documentation provided by each center to the implementation of audit visits that are going to be the basis on which the decision whether or not to issue a certificate will be taken.

\section{Recommendation 7}

Postmarketing data collection for the long-term safety and efficacy of all products should be mandatory. Every country should establish a national patient registry for hemophilia and other inherited bleeding disorders, covering all treatment modalities and patient-relevant outcomes.

From the beginning, the Kreuth Initiative for optimal use of blood products has been producing recommendations for hemophilia treatment, ${ }^{4}$ but so far the issue of data collection has not been addressed. Clinical registries are important tools, particularly in the context of rare diseases such as hemophilia characterized by a limited number of patients available for clinical trials. Registries may also help to collect long-term real-life data on the usage of products and patient-relevant treatment outcomes, thus providing valuable safety information. Registries should be set up in each European country on a national basis, and ideally should include all patients with inherited bleeding disorders.

Although recent EMA guidelines set standards for clinical studies, ${ }^{24}$ the design and conduct of the currently available studies are characterized by several differences which prevent comparative data analysis. Registries may provide relevant complementary real-life data across a variety of products. ${ }^{25}$ Alongside the reporting obligations laid down by the pharmacovigilance legislation, registries should also collect comprehensive postmarketing safety information on all treatment modalities.

\section{Recommendation 8}

Dedicated data governance, evaluation and reporting should be implemented with adequate sustainable financial support under public control. Core data elements as recommended by the European Medicines Agency (EMA) $)^{24,26}$ and minimal dataset for post-registration surveillance should be implemented according to the communication from the International Society on Thrombosis and Haemostasis (ISTH) Scientific and Standardization Subcommittee (SSC) on Factor VIII, Factor IX and Rare Coagulation Disorders. ${ }^{27}$

Although there are a number of ongoing registries in Europe, their organization and status, as well as the amount and quality of collected data, is quite variable. ${ }^{28}$ There are various models of registries, and promoters may be patient organizations, scientific societies, networks of treatment centers or government institutions. Important questions need to be answered and solutions found for the problems related to each registry. How is the collection of data organized (preferably as user-friendly online portals)? How are data integrity and quality assured? Who owns the data? Who performs which analysis? How and by whom are results reported and eventually published? Additional challenges may also be presented by some degree of reluctance from hemophilia caregivers to share their patients' data and to make the effort needed to enter the data into a registry. To tackle these challenges, the German Transfusion Act was updated to include mandatory adherence to the German Hemophilia Register Participation agreement. The organization of registries is a complex affair, and requires thorough planning, and substantial and sustainable support. Given the importance and relevance of the data for the scientific evaluation of hemophilia treatment by regulatory agencies, and the particular sensitivity of patient data, registries and their funding need to be under public control. In order to enable meaningful evaluation and comparative analysis, a minimum set of common data elements should be included, as recently recommended by the EMA. ${ }^{26}$ Detailed guidance concerning the data set required for post-registration surveillance was also previously communicated by the ISTH Scientific and Standardization Committee on Factor VIII, Factor IX and Rare Coagulation Disorders. ${ }^{27}$

\section{Recommendation 9}

Collaboration at the European level should be encouraged, by strengthening and harmonizing existing registries in order to facilitate pooling and comprehensive evaluation of data.

Greater collaboration is essential in order to have more data available for evaluation, and to enable data pooling and a comprehensive evaluation of data across all treatment modalities at a European level. Although the performance, co-operation and outputs of the registries currently operative in Europe are not satisfactory ${ }^{28}$ the way forward is not to advocate a single new, pan-European super-registry, but to strengthen and harmonize those already existing, as well as to encourage the establishment of common tools and strategies for their optimal use. This is an important and ambitious task which requires the support of all stakeholders, including patients, those responsible for their treatment, and academia, regulatory authorities and policymakers involved in health care.

\section{Recommendation 10}

Data collection should include direct reporting from patients using appropriate electronic industry-independent tools. Its use should be supported by education, user-friendly applications, and positive feedback to the patients.

The ultimate success of any kind of hemophilia registry depends on the participation and commitment of the caregivers and the willingness of patients to provide their data. In particular, the accuracy and completeness of the collected data are closely related to the tools made available for reporting them to the registry. Data collection by direct reporting from patients through electronic tools is an attractive approach, provided any bias resulting from industry-developed applications is excluded, and treating physicians supervise its management and exercise quality control. Appropriate information and education about the background, instruments and goals of each registry, and provision of user-friendly applications are particularly 
important. It is also important to provide patients with a positive feedback to underline the value of their contributions.

\section{Recommendation 11}

Clinical studies should be performed to provide the best possible evidence needed for regulatory authorities, health technology assessment (HTA) bodies, academia and healthcare providers.

Clinical trials conducted during the drug licensing process provide the most important information for predicting the efficacy and value of new medicines. While clinical trial data are the gold-standard of the evidencebased efficacy needed by regulatory authorities and health care providers, their usefulness often has limitations with respect to HTA and determining their value within the framework of cost-effectiveness studies, primarily carried out in the interest of the decision-making processes of payers. The purpose of most clinical trials is to test hypotheses about the efficacy and side effects of medications compared against placebo or a selected comparator therapy. HTA aims to answer questions about how these findings can be translated into clinical practice and change the standard of care, but also on how to estimate costeffectiveness, with the goal of informing decisions intended to ensure value for money. Most clinical trials often do not focus on end points such as the relevant patient outcomes requested by HTA bodies and/or payers. These limitations have acquired greater importance since regulatory authorities introduced new initiatives such as adaptive licensing in order to accelerate access to innovative treatments, particularly in the context of orphan or rare diseases. Smaller and shorter trials may be able to provide evidence faster, but they will fail to generate enough information for patient assessment of relevant outcomes and for cost-effectiveness. To minimize these gaps of knowledge, and to provide sufficient evidence to promote effective HTA and cost effectiveness analysis, clinical trial designs for innovative therapies should be optimized to reduce uncertainty, eliminate bias, decrease costs, and accelerate patient access.

\section{Recommendation 12}

A process to reach better agreement on relevant indicators and methods should be started in order to meet the needs of regulatory authorities, HTA bodies, academia and healthcare providers, that have different foci, responsibilities and requirements.

There is a strong need for a multidisciplinary consensus on measurable, patient-relevant outcome indicators which reflect the benefits of new and innovative hemophilia therapies. Consensus on the choice of outcome assessment instruments should also be reached in order to allow for a more effective combination of data from different sources. These goals will be necessary to obtain harmonized and transparent decision-making processes. Another subject for discussion should be whether outcomes should be collected in clinical trials or if data from other sources such as observational studies including registries should be complementary sources to assess benefit within hemophilia care. Furthermore, a minimum set of methods for outcome determination would help the different decision makers to make their assessment. Because hemophilia is a chronic rare disease, and concrete patientrelevant outcomes such as avoidance of joint damage cannot be evaluated at the time of market entry of innovative therapies, it is important to identify appropriate surrogate indicators and agree on statistical methods for prognostic calculations in order to provide estimates also on mediumto long-term consequences.

\section{Recommendation 13}

A dialogue with agencies and academia should be started in order to reach understanding of relevant outcomes and to ensure comprehensive and consistent reporting.

Health Technology Assessment bodies and/or payers request information on patient-relevant clinical end points in order to provide the evidence of therapeutic benefit of new or innovative therapies compared to the standard of care. These hard end points are mainly morbidity, mortality and quality of life, but these only partially reflect the benefits associated with innovative hemophilia therapies. ${ }^{29}$ Although mortality in hemophilia was very high before the introduction of replacement therapy, nowadays it is almost comparable to that of the general population, so it cannot be seen as an appropriate outcome to be measured at the time of drug evaluation and market entry. ${ }^{30}$ Repeated joint bleeds cause morphological changes and in the long run lead to arthropathy; but many years of follow up would be required to assess hemophilic arthropathy and its consequences. Multifactorial influences lead to individual variations in a small patient population such as hemophilia pertaining to the bleeding phenotype, development of arthropathy, and inhibitory antibodies. Thus, the measurement of health-related quality of life (HRQoL) might be a reasonable indicator of the long-term outcome and effectiveness of the therapeutic intervention chosen. It can also be converted into utility values to enable the assessment of the quality-adjusted life years gained, and to calculate cost-effectiveness. Both hemophilia disease-specific instruments and generic instruments are available and should be implemented as a standard procedure.

\section{Acknowledgments}

Co-sponsoring by Paul Ehrlich Institut, the LudwigMaximilian University of Munich and the European Directorate for the Quality of Medicines and Healthcare. Thanks to Isabella Garagiola, Ph.D. from Angelo Bianchi Bonomi Hemophilia and Thrombosis Center for helping us in the preparation of the manuscript and to all participants and delegates who actively participated in the discussion with the three working groups, providing their own input and helping to produce this Kreuth V European consensus for treatment of hemophilia.

\section{References}

1. Schramm W, von Auer F, Delaney F, Seitz R. Blood Safety in the European Community: An Initiative for Optimal Use. Wildbad Kreuth, 20-22 May, 1999. Conference
Proceedings. ISBN 3-00-005705-6.

2. Berger K, Klein HG, Seitz R, Schramm W, Spieser JM. The Wildbad Kreuth initiative: European current practices and recommendations for optimal use of blood components. Biologicals. 2011;39(3):189-193.

3. Giangrande P, Seitz R, Behr-Gross ME, et al.
Kreuth III: European consensus proposals for treatment of haemophilia with coagulation factor concentrates. Haemophilia. 2014;20 (3):322-325

4. Giangrande PLF, Peyvandi F, O'Mahony B, et al. Kreuth IV: European consensus proposals for treatment of haemophilia with coagula- 
tion factor concentrates. Haemophilia. 2017;23(3):370-375.

5. Council of Europe, Committee of Ministers, Resolution CM/Res(2017)43 on principles concerning haemophilia therapies (replacing Resolution CM/Res(2015)3).

6. Manco-Johnson MJ, Abshire TC, Shapiro $\mathrm{AD}$, et al. Prophylaxis versus episodic treatment to prevent joint disease in boys with severe haemophilia. $N$ Engl J Med. 2007;357(6):535-544.

7. Berntorp E, Astermark J, Björkman S, et al. Consensus perspectives on prophylactic therapy for haemophilia: summary statement. Haemophilia. 2003;9(Suppl 1):1-4.

8. Fischer K, Astermark J, van der Bom JG, et al. Prophylactic treatment for severe haemophilia: comparison of an intermediate-dose to a high-dose regimen. Haemophilia. 2002;8 (6):753-760.

9. Peyvandi F, Garagiola I, Young G. The past and future of haemophilia: diagnosis, treatments, and its complications. Lancet. 2016;388(10040):187-197

10. Berntorp E, Andersson NG. Prophylaxis for hemophilia in the Era of extended Half-Life Factor VIII/Factor IX Products. Semin Thromb Hemost. 2016;42(5):518-525.

11. Peters R, Harris T. Advances and innovations in haemophilia treatment. Nat Rev Drug Discov. 2018;17(7):493-508.

12. Oldenburg J, Mahlangu JN, Kim B, et al. Emicizumab prophylaxis in hemophilia A with inhibitors. N Engl J Med. 2017;377 (9):809-818.

13. Mahlangu J, Oldenburg J, Paz-Priel I, et al. Emicizumab prophylaxis in patients who have hemophilia A without inhibitors. N Engl J Med. 2018;379(9):811-822.

14. Stephensen D, de Kleijn P, Matlary RED, et al. EAHAD Physiotherapists Committee. Scope of practice of haemophilia physiotherapists: A European survey. Haemophilia. 2019;25(3):514-520

15. Collins PW. Personalized prophylaxis. Haemophilia. 2012;18(Suppl. 4):131-135.

16. Hazendonk HCAM, van Moort I, Mathôt RAA, et al. Setting the stage for individualized therapy in hemophilia: What role can pharmacokinetics play? Blood Rev. 2018;32 (4):265-271.

17. Collins PW, Blanchette VS, Fischer K, et al. Break-through bleeding in relation to predicted factor VIII levels in patients receiving prophylactic treatment for severe hemophilia A. J Thromb Haemost. 2009; 7(3):413-420.

18. Kitchen S, Tiefenbacher S, Gosselin R Factor Activity Assays for Monitoring Extended Half-Life FVIII and Factor IX Replacement Therapies. Semin Thromb Haemost. 2017:43(3):331-337.

19. Tripodi A, Chantarangkul V, Novembrino C, Peyvandi F. Advances in the treatment of hemophilia: Implications for laboratory testing. Clin Chem. 2019;65(2):254-262.

20. Nogami K, Soeda T, Matsumoto T, Kawabe Y, Kitazawa T, Shima M. Routine measurements of factor VIII activity and inhibitor titer in the presence of emicizumab utilizing anti-idiotype monoclonal antibodies. J Thromb Haemost. 2018;16(7):1383-1390.

21. Teichman J, Chaudhry HR, Sholzberg M. Novel assays in the coagulation laboratory: a clinical and laboratory perspective. Transfus Apher Sci. 2018:57(4):480-484.

22. Müller J, Pekrul I, Pötzsch B, Berning B, Oldenburg J, Spannagl M. Laboratory Monitoring in Emicizumab-Treated Persons with Hemophilia A. Thromb Haemost.
2019;119(9):1384-1393.

23. Collins PW, Liesner $\mathrm{R}$, Makris $\mathrm{M}$, et al. Treatment of bleeding episodes in haemophilia A complicated by a factor VII inhibitor in patients receiving Emicizumab. Interim guidance from UKHCDO Inhibitor Working Party and Executive Committee. Haemophilia. 2018;24(3):344-347.

24. Guideline on the clinical investigation of recombinant and human plasma-derived factor VIII products. EMA/CHMP/BPWP/ 144533/2009 rev. 2

25. Keipert C, Jonker CJ, van den Berg HM Hilger A. Clinical trials and registries in haemophilia: Opponents or collaborators? Comparison of PUP data derived from different data sources. Haemophilia. 2018;24 (3):420-428.

26. Report on Haemophilia Registries Workshop 8 June 2018. EMA/487643/2018. https://www.ema.europa.eu/en/documents/report/report-haemophilia-registriesworkshop_en.pdf

27. Peyvandi F, Makris M, Collins $\mathrm{P}$, et al Minimal dataset for post-registration surveillance of new drugs in hemophilia: communication from the SSC of the ISTH. Thromb Haemost. 2017;15(9):1878-1881.

28. Keipert C, Hesse J, Haschberger B, et al. The growing number of hemophilia registries: Quantity versus quality. Clin Pharmacol Ther. 2015;97(5):492-501.

29. Berger KC, Feldman BM, Wasserman J, et al. Securing reimbursement for patient centered haemophilia care: major collaborative efforts are needed. Haematologica. 2016;101(3):266-268.

30. Mannucci PM. Hemophilia therapy: the future has begun. Haematologica. 2020;105 (3):545-553 